\title{
EFFECT OF SYNTHESIZED BIS-GMA AND UDMA NANOFIBERS ON CUSPAL FLEXURE, MICROHARDNESS, WEAR, SURFACE ROUGHNESS AND COLOR STABILITY OF EXPERIMENTAL RESIN-COMPOSITES
}

\author{
Samy M. El-Safty ${ }^{*}$ and El-Refaie S. Kenawy ${ }^{* *}$
}

\begin{abstract}
Objective: To study the cuspal flexure (CF), Vickers microhardness (HV), three-body wear (W), surface roughness (SR) and color stability (CS) of experimental resin-composites reinforced with Bis-GMA and UDMA nanofibers synthesized by electrospinning technique.

Materials and methods: Bis-GMA (Bis-GMA+TEGDMA+PEGDMA) and UDMA (UDMA+PEGDMA) nanofibers were synthesized by wet electrospinning technique and characterized by Scanning Electron Microscopy (SEM) and Fourier Transform Infrared Spectroscopy (FTIR). After being ball-milled to a nanoscale size $(<100 \mathrm{~nm})$ in a ball-milling machine (Retsch - PM 400 , Haan, Germany), these nanofibers were added to a prepared experimental resin-composite. This study was divided, according to the percent and type of added nanofibers, into eight groups ( $n=10$ /group except for color stability $n=11$ ): one control group, three groups reinforced with 7,11 and $15 \mathrm{wt} \%$ Bis-GMA nanofibers and four groups reinforced with 7, 11, 15 and $20 \mathrm{wt} \%$ UDMA nanofibers. For each of these groups, CF, HV, W, SR and CS were studied. CF was studied by the digital image correlation method which analyzes the displacement of an object using a USB digital microscope with a built-in camera (COMET xS, Steinbichler Optotechnik GmbH, Neubeuern, Germany). HV was measured according to the ASTM E-384:1999 using a Digital Display Vickers Microhardness Tester (ZHV $\mu$ Micro Vickers Hardness Tester, Indentec Hardness Testing, Zwick Roell Co., Ltd. Atlanta, USA). W testing was carried out according to ISO/TS 14569:2001 using a custom-made cusp-on-disc sliding machine. Measurement of SR was performed according to ISO 25178-2:2012 and CS testing was carried out according to the regulations of ISO 4049; 2009 using a portable reflective spectrophotometer (X-Rite, model RM200QC, Neu-Isenburg, Germany). Data were collected and submitted to One-way ANOVA and post-hoc Tukey and Dunnett's T3 tests with the significance level set at $(\mathrm{p} \leq 0.05)$.
\end{abstract}

* Lecturer, Department of Dental Biomaterials, Faculty of Dentistry, Tanta University, Tanta, Egypt.

** Professor, Department of Chemistry, Director of Technology Transfer office, Faculty of Science, Tanta University, Tanta, Egypt. 
Results: Pure forms of Bis-GMA (70-100 nm) and UDMA (50-100 nm) nanofibers were confirmed by SEM and FTIR. One-way ANOVA revealed significant differences between studied groups for CF, HV, W and SR ( $p=0.001)$ but not for CS $(\mathrm{p}=0.068)$. For both types of nanofibers, compared to the control group, reinforced groups showed lower CF, HV and SR and greater W results. For CS, there was no consistent trend of results of experimental groups that were comparable to that of control group. Within the range of added nanofibers (7-20 wt\%), Bis-GMA nanofibers exhibited more favourable $\mathrm{HV}$ and $\mathrm{W}$ results than UDMA nanofibers, while the reverse was the case for CF and SR. Both nanofibers exhibited comparable CS results.

Clinical Significance: A resin-composite reinforced with such nanofibers showing improved $\mathrm{CF}$ and unfavorably lower HV and more W should be used as a base layer and covered with a layer of another resin-composite with enhanced surface properties.

KEYWORDS: Nanofibers; Bis-GMA; UDMA; Experimental Resin-Composite; Cuspal Flexure; Microhardness; Wear; Surface roughness and Color stability.

\section{INTRODUCTION}

The ability to endure static and cyclic mechanical loading, as well as the exposure to organic solvents while in function, determine, to a great extent, the clinical performance of dental resin-composites. Their physical-chemical properties are majorly influenced by the organic content, as it influences the degree of conversion and the structure of the resultant polymer ${ }^{[1,2]}$. Most commercial resincomposites have their organic matrix made up of co-polymerization of dimethacrylate monomers. These molecules are characterized by particular features that define their reactivity and mobility during polymerization reaction and determine the degree of conversion and crosslink density of the polymer formed ${ }^{[3,4]}$.

The dimethacrylates Bis-GMA (2, 2-bis-[4(2-hydroxy-3 methacryloyloxypropoxy) phenyl] propane) and UDMA (1,6-bis(methacryloxy-2ethoxycarbonylamino)-2,4,4-trimethylhexane) are widely used as the base monomers in the formation of the polymeric matrices of dental resincomposites. These two monomers are comparable in size but have different chemical structural characteristics that affect critical properties such as viscosity, degree of conversion, polymerization shrinkage, water uptake, refractive index and optical matching with reinforcing fillers, physico- chemical and mechanical properties. The capacity to form physical crosslinks through hydrogen bonding that can enhance the basic strength and modulus of their dimethacrylate-based polymers is a common feature of Bis-GMA and UDMA. This crosslinking is mainly achieved by carbon-carbon covalent bonding, especially in the form of chemical crosslinks of the network structure ${ }^{[5]}$.

The filler part of resin-based restorative materials plays a crucial role in enhancing their physical/mechanical properties ${ }^{[6-8]}$. Reinforcing resin-composites with short or long fibers has been reported in the literature for many decades ${ }^{19-}$ ${ }^{11}$. Fiber fillers are superior to particulate fillers in many respects. These include greater resistance to fracture because of stress transfer from the matrix to the fibers depending on their length and diameter ${ }^{[12,13]}$. The high aspect ratio, i.e. ratio between the length and diameter $(1 / \mathrm{d})$, of the fibers significantly contributes to the reinforcing efficiency of fibers [14]. In addition, the greater surface area of the fiber fillers compared to the particulate fillers provides better bonding with the resin matrix ${ }^{[15]}$. Inhibition of crack initiation and propagation is one more critical advantage of fiber fillers ${ }^{[16]}$.

Recently, electrospinning has been applied as a suitable technology for the production of polymeric fibers at the scale of nanometer ${ }^{[17]}$. The process 
starts with generating a high voltage between the polymer solution (negatively charged) contained in a syringe feeder and a metallic collector (positively charged). The resulting electrostatic force causes the polymer solution to elongate and produce ultrafine fibers (nanometer-sized) on top of the collector in the form of a randomly oriented fibrous mat with a large surface area to volume ratio ${ }^{[18]}$.

Polymerization shrinkage of resin-composites has been considered one of the main drawbacks of such materials ${ }^{[19]}$. This is because of the direct relationship between stresses generated during polymerization and the integrity of the restorationtooth margins ${ }^{[20]}$. These stresses may cause cuspal flexure and bonding failure between resin-composite filling and tooth structure. Cuspal flexure is defined as a biomechanical phenomenon that takes place as result of the interaction between polymerization shrinkage stress of the resin-composite and the compliance of the cavity wall ${ }^{[21]}$. The volumetric shrinkage of resin-composite materials can be, as reported by Giachetti et al. ${ }^{[22]}$, and Labella et al. ${ }^{[23]}$, within the range of $2-6 \%$.

Hardness of resin-composite materials is a good indicator of their clinical performance. It reflects, indirectly, the extent of polymerization of the material ${ }^{[24]}$. It is majorly related to the filler fraction of the material ${ }^{25,26]}$. Hardness testing has been valuable to predict the wear resistance of a material and its liability to abrade or to be abraded by the opposing tooth structure and materials ${ }^{[27,28]}$. In small and medium sized dental cavities, wear of current resin-composite restorations is no longer a big issue. In larger posterior cavities, however, these restorative materials are still at risk of extensive wear, particularly in patients with abnormal habits like bruxism ${ }^{[29,30]}$. It was reported that wear of resin-composites is strongly related to the material type and the presence of a third medium ${ }^{[31-33]}$. Introduction of nano-sized filler particles to the resin-composite formulation greatly enhanced its wear resistance. While maintaining good handling characteristics, the smaller particle size enables higher filler loading and better wear resistance ${ }^{[34]}$.

Surface smoothness of a restorative material is a very essential practice when doing a restoration. It is important to avoid rough surface to prevent food retention on the restoration surface, and hence, its color change. Additional harmful consequences of a rough surface is plaque formation, gingival inflammation and recurrent caries ${ }^{[35,36]}$. Surface roughness has been defined as the fine irregularities existing on a material's surface that usually results from the manufacturing process or because of the characteristics of the material itself ${ }^{[37]}$. It was reported that a mean roughness of $0.2 \mu \mathrm{m}$ is considered the critical threshold value for bacteria retention on the restoration surface ${ }^{[38]}$. A perfect resin-composite restoration, particularly in anterior teeth, depends - in part - on the color-matching between the restorative material and the tooth being restored. However, there are many variables threatening the color stability of such restorative materials. The composition of the resin matrix and its hydrophilicity are critical factors in color change ${ }^{[39]}$. The filler type, particle size and distribution play a role in that respect ${ }^{[40]}$. Inadequate polymerization, staining from diet and hygiene habits can effectively influence the restoration color as well ${ }^{[41]}$.

The objectives of our study were: firstly, synthesis of Bis-GMA and UDMA nanofibers by electrospinning technique for the reinforcement of an experimental resin-nano-composite. Secondly, characterization of the synthesized nanofibers by scanning electron microscopy (SEM) and Fouriertransform infra-red spectroscopy (FTIR). Thirdly, comparing the effect of added Bis-GMA nanofibers with that of UDMA nanofibers on some properties of experimental resin-composite: cuspal flexure, Vickers microhardness, wear, surface roughness and color stability. Our null hypotheses were: i) the electrospun organic nanofibers will have 
no significant effect on the studied properties of the experimental resin-composites and ii) there will be no difference between the effect of BisGMA nanofibers and that of UDMA nanofibers on the examined properties of experimental resincomposites.

\section{MATERIALS AND METHODS}

2.1. Materials: Information of percents and supplier of the materials and chemical ingredients used in this study are listed in Table 1.

\subsection{Methods:}

\section{Preparation of nanofibers by electrospinning:}

In the present study, preparation, characterization and ball-milling of Bis-GMA and UDMA nanofibers were carried out in the same way described in a previous study conducted by the authors ${ }^{[42]}$.

\section{Formulation of experimental resin-composites:}

Preparation and silanation of the filler nanoparticles, preparation of the resin part and formulation of experimental resin-composites were performed according to the previous study ${ }^{[42]}$ as well. This filler mixture was divided according to the study groups to be investigated (eight groups: one control and seven experimental). One group was prepared without nanofibers. Other three groups were formed by adding Bis-GMA nanofibers at the ratio of 7,11 and $15 \mathrm{wt} \%$ at the expense of the nanoparticles. More four groups were formulated by addition of UDMA nanofibers at the ratio of 7 , 11,15 and $20 \mathrm{wt} \%$ at the expense of nanoparticles. The added nanofibers were thoroughly mixed with the nanoparticles in a mechanical stirrer (5040001 RW28, Atlanta, USA) at $50 \mathrm{rpm}$ for $6 \mathrm{~h}$ at room temperature to ensure even distribution of the nanofibers throughout the fillers. The photoinitiator (camphorquinone) and co-initiator (4EDMAB) were mixed in a proportion of 1: $1 \mathrm{wt} \%$ in the mechanical stirrer at $10 \mathrm{rpm}$ for $1 \mathrm{~h}$ at room temperature to form the photo-activation system. Finally, each group of the experimental resin-composite was prepared in the proportions of $27 \mathrm{wt} \%$ organic matrix, $72 \mathrm{wt} \%$ fillers and $1 \mathrm{wt} \%$ photo-activation system. Mixing process was achieved in a centrifugal mixing device (Speed-Mixer, DAC 150 FVZK, Hauschild Engineering, Germany).

\section{Grouping:}

This study was divided into one control group and seven experimental groups according to the type and percent of the added nanofibers as follows:

TABLE (1) Information and percentages of chemical ingredients used in preparing the experimental resincomposite and electrospun organic (Bis-GMA and UDMA) nanofibers.

\begin{tabular}{|l|l|}
\hline \multicolumn{1}{|c|}{ Chemical Ingredients } & \multicolumn{1}{c|}{ Supplier } \\
\hline A) Resin 27 wt\%: & \\
1) Bisphenol A diglycidyl ether dimethacrylate (Bis-GMA). & \\
2) Tri (ethylene glycol) dimethacrylate (TEGDMA) & \\
3) Urethane Dimethacrylate (UDMA). & \\
4) Bisphenol A polyethethylene glycol diether dimethacrylate (Bis-EMA). & Sigma Aldrich, St Louis, \\
5) Polyethylene Glycol Dimethacrylate (PEGDMA). & Missouri Ltd, USA \\
B) Nano-fillers 72 wt\%: <50 nm nanoparticles and 0.3 $\mu$ m loosely agglomerated clusters & \\
of silica and zirconia. & \\
C) Initiator and accelerator 1 wt\%: & \\
1) Camphorquinone (CQ). & \\
2) Ethyl-4-(N,N'-dimethylamino) benzoate (4EDMAB). & \\
D) Silane: 3-methacyloxypropyltrimethoxysilane. & \\
\hline
\end{tabular}


Group I: Experimental composite without nanofibers.

Group II: Experimental composite reinforced with $7 \mathrm{wt} \%$ Bis-GMA nanofibers.

Group III: Experimental composite reinforced with 11 wt $\%$ Bis-GMA nanofibers.

Group IV: Experimental composite reinforced with $15 \mathrm{wt} \%$ Bis-GMA nanofibers.

Group V: Experimental composite reinforced with 7 wt\% UDMA nanofibers.

Group VI: Experimental composite reinforced with $11 \mathrm{wt} \%$ UDMA nanofibers.

Group VII: Experimental composite reinforced with $15 \mathrm{wt} \%$ UDMA nanofibers.

Group VIII: Experimental composite reinforced with 20 wt $\%$ UDMA nanofibers.

\section{Studied properties:}

The properties evaluated in the present study were: cuspal flexure $(\mathrm{CF})$, Vickers microhardness $(\mathrm{HV})$, three-body wear (W), surface roughness (SR) and color stability (CS). For each property, ten specimens were prepared and tested for each group ( $n=10)$ except for CS ( $n=11$ per group). To ensure proper curing of the tested specimens, a calibrated radiometer system (MARC Blue-Light Analytics Inc., Halifax, NS, Canada) was used to verify the irradiance of the light curing unit periodically, after each group.

\section{Cuspal flexure (CF)}

A total of eighty maxillary premolars were collected from Oral surgery and orthodontic departments according to Research Ethics Committee, Faculty of Dentistry, Tanta University. The purpose of the present study was explained to the patients and informed consents were obtained. Teeth were thoroughly cleaned and stored in $0.5 \%$ chloramine solution at $23 \pm 1{ }^{\circ} \mathrm{C}$ until they were submitted to cavity preparation. Preparation of teeth for $\mathrm{CF}$ testing was conducted according to previous published studies [43, 44]. Using a high speed handpiece and a parallel diamond bur (Miltex RA \#556, Miltenberg Inc, New York, USA) and under water cooling each tooth was prepared for a large standardized Class II mesio-occlusal-distal (MOD) cavity. The width of the proximal box was twothirds of the bucco-palatal width (BPW). A gingival seat of nearly $1 \mathrm{~mm}$ was prepared and extended above the cemento-enamel junction (CEJ) at the cervical aspect. The occlusal isthmus was prepared to the half of the BPW and the cavity depth at the occlusal isthmus was standardized to $3 \mathrm{~mm}$. for all specimens, the width of occlusal box was $4 / 5$ of the intercuspal width. The cavo-surface margins were prepared at $90^{\circ}$ and all the internal line angles were rounded. Periodontal probe was used to verify preparation dimensions of the prepared cavities.

After cavity preparation, the teeth were mounted in an auto-polymerizing acrylic resin (PMMA; Esschem Co., PA, USA).The CF for all groups was measured using the digital image correlation method which analyzes the displacement of an object using a USB digital microscope with a built-in camera (COMET xS, Steinbichler Optotechnik GmbH, Neubeuern, Germany). Two reference points were selected on the buccal and palatal cusps of each tooth. After that, the mounted teeth were scanned using an optical scanner (COMET xS, Steinbichler Optotechnik GmbH, Neubeuern, Germany) before restoration and the initial length between the reference points $\left(\mathrm{L}_{0}\right)$ was recorded.

The eighty premolar teeth were divided into eight groups $(n=10)$; control group and seven experimental groups according to the type and percent of added nanofibers. The tooth surfaces were etched and prepared for bonding using a bonding system (Adper $^{\mathrm{TM}}$ Single Bond Plus Adhesive Refill, 51102, $3 \mathrm{M}^{\mathrm{TM}}$, ESPE, USA) according to the manufacturer's instructions. The experimental resin-composite was packed inside the cavity in horizontal increments with $2 \mathrm{~mm}$ thickness of each. Each increment was 
light-cured for $20 \mathrm{~s}$ using a light curing unit (Elipar

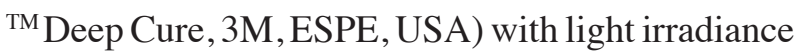
of $1200 \mathrm{~mW} / \mathrm{cm}^{2}$. Each restored tooth was scanned $10 \mathrm{~min}$ after restoration. The distance between the reference points $\left(\mathrm{L}_{\text {Final }}\right)$ after curing (deformation; deformed image) was recorded. The cuspal flexure was calculated as the difference between the final and initial measurements, $\Delta \mathrm{L}=\mathrm{L}_{\text {Final }}-\mathrm{L}_{0}$. Percentage of cuspal flexure was calculated by: cuspal flexure $\%=\left(\Delta \mathrm{L} / \mathrm{L}_{0}\right) * 100$.

\section{Vickers microhardness (HV)}

Microhardness testing was carried out according to the ASTM E-384:1999 [45] Specimens were prepared in a cylindrical stainless steel mold (5 $\mathrm{mm}$ diameter $\times 2 \mathrm{~mm}$ thickness). Mold was put on a glass slide covered with Mylar strip and a separating medium was applied to its inner walls using a brush. The mold was slightly overfilled with the composite material and the excess was then extruded by applying another glass slab covered with Mylar strip pressed firmly. Each specimen was then cured from the top surface only for $20 \mathrm{~s}$ using an LED light curing unit (Elipar ${ }^{\mathrm{TM}}$ Deep Cure $3 \mathrm{M}$ ESPE, USA) under a standard curing mode. The light curing unit had a $10 \mathrm{~mm}$ tip with an output irradiance of $1200 \mathrm{~mW} / \mathrm{cm}^{2}$ and wavelength range of 430-480 $\mathrm{nm}$. Immediately after cure, the specimen was gently pushed out from the mold. The top surface of each specimen was ground with a series of silicon carbide $(\mathrm{SiC})$ abrasive papers $(600,1200$ and 2000-grit) and polished with diamond paste ( $3 \mu \mathrm{m}$ and $1 / 4 \mu \mathrm{m})$ to produce smooth and uniform surface. The top surface of each specimen was marked with a permanent marker.

Surface microhardness of the specimens was tested using a Digital Display Vickers Microhardness Tester $(\mathrm{ZHV} \mu$ Micro Vickers Hardness Tester, Indentec Hardness Testing, Zwick Roell Co., Ltd. Atlanta, USA) with a Vickers diamond indenter and a 20x objective lens. A load of $50 \mathrm{gm}$ was applied to the top surface of the specimen for $15 \mathrm{~s}$. Each specimen was subjected to five indentations, equallyspaced, over a circle. Care was taken to make the indentation not closer than $1 \mathrm{~mm}$ to the adjacent indentations or the margin of the specimen. The diagonals length of the indentations was measured with a built-in scaled microscope. Micro-hardness was obtained using the following equation:

$$
\mathrm{HV}=1.854 \mathrm{P} / \mathrm{d}^{2}
$$

Where, $H V$ is Vickers microhardness in $\mathrm{HVN}=\mathrm{Kg} / \mathrm{mm}^{2}, P$ is the load in $\mathrm{Kg}$ and $d$ is the length of the diagonals in $\mathrm{mm}$.

\section{Three-body wear (W)}

Three-body wear testing was carried out according to ISO/TS 14569:2001 ${ }^{[46]}$. Specimens were prepared in a stainless-steel cylindrical mold of $8 \mathrm{~mm}$ diameter and $2 \mathrm{~mm}$ thickness. The resincomposite material was applied to slightly overfill the mold cavity seated on a glass plate covered with Mylar strip and pressed flush under another glass plate prior to $20 \mathrm{~s}$ light activation using an LED light curing unit (Elipar ${ }^{\mathrm{TM}}, 3 \mathrm{M}$ ESPE, USA) that had a $10 \mathrm{~mm}$ tip with an output irradiance of 1200 $\mathrm{mW} / \mathrm{cm}^{2}$ and wavelength range of $430-480 \mathrm{~nm}$. Immediately after curing, specimens were stored in water at $37^{\circ} \mathrm{C}$ for 1 week. The excess composite was removed on wet $600 \#, 1200 \#, 1500 \#$ and $4000 \#$ silicon carbide abrasive paper, then specimens were polished sequentially with a complete series of Soflex polishing discs (3M ESPE, St Paul, MN, USA). For standardization, just a single operator performed the polishing procedure using a lowspeed handpiece at approximately 4,000-5,000 rpm. The polished surfaces were water-rinsed with an airwater syringe for $60 \mathrm{~s}$, to remove any surface debris left and then were air-dried for $30 \mathrm{~s}$.

Prior to wear testing, each specimen was optically analyzed using a USB Digital microscope with a built-in camera (COMET xS, Steinbichler Optotechnik GmbH, Neubeuern, Germany) connected to an IBM compatible personal computer using a magnification fixed at 100 -fold at $5 \mu \mathrm{m}$ steps 
along the z-axis. Each specimen was individually placed in a positioning platform that allows further placement of the specimen in the same position for the post-test measurements. The original profile of the specimens was traced for post-test analysis of contour changes.

Human molars, extracted according to Research Ethics Committee, Faculty of Dentistry, Tanta University were used in this study. The purpose of the present study was explained to the patients and informed consents were obtained. Molars were sectioned using a low-speed diamond saw (Isomet, Buehler, Illinois, USA) and cast into a knob on the heads of flat-head screws by means of metallographic epoxy. This knob was dressed on a lathe, by means of a greenstone in a pivoting fixture, into a $10-\mathrm{mm}$ diameter spherical shape. The enamel was sanded with 400- and 600-grit SiC abrasive paper and polished with 1000-grit $\mathrm{SiC}$ and 5- $\mu \mathrm{m}$ aluminum oxide to get a surface roughness of $0.75 \mu \mathrm{m}{ }^{[47]}$. The enamel surface of the attached molar was checked periodically during testing. If a great amount of enamel was worn away, the molar was replaced with a new one for standardized wear results.

Wear-testing device is a custom-made cuspon-disc sliding machine. The surface of resincomposite specimen was loaded $(8 \mathrm{~N})$ at $15^{\circ}$ angulation (ISO/TS14569-2), the sliding path length was $3.7 \mathrm{~mm}$, then the molar cusp was lifted and returned to the starting position for the next cycle. Each specimen was cycled 50,000 times, an amount which roughly produces the same amount of wear that may occur during a six-month period of in vivo service ${ }^{[48]}$. Cycling was performed at $1 \mathrm{~Hz}$, which approximates the measurements of chewing rates found in numerous literature studies ${ }^{[49]}$.

The abrasive slurry used for the wear testing consisted of $3 \mathrm{~g}$ of poppy seeds, $1.5 \mathrm{~g}$ of PMMA beads (Lucitone 199, Dentsply International Inc. Chicago, USA, average bead size $40 \mu \mathrm{m}$ ), and 15 $\mathrm{ml}$ of deionized water at $23 \pm 2{ }^{\circ} \mathrm{C}$. This slurry was prepared according to De Gee et al. ${ }^{[4]}$ to produce a clinically relevant environment for three-body wear testing. Ten mg of thymol was added to the mixture to reduce spoilage. The seeds were ground with 100 strokes in a mortar and pestle to increase miscibility. Three $\mathrm{ml}$ of well-stirred slurry was added to each of the chambers. Cycling was begun within an hour after the slurry was mixed. Each specimen was mounted in an aluminum container filled with the abrading slurry. The slurry was renewed after each 10,000 cycles. Testing was done at ambient laboratory atmosphere. After being cycled, the specimens were removed from the wear chambers and rinsed in water. Each specimen was reanalyzed and quantitative wear was determined as loss of substance in $\mathrm{mm}$.

\section{Surface roughness $(\mathrm{SR})$}

Surface roughness testing was performed according to ISO 25178-2:2012 ${ }^{[50]}$. Specimen preparation, curing, polishing and cleaning was done exactly as that for the three-body wear testing. The optical method was used to measure average surface roughness. Specimens were photographed using a USB Digital microscope with a built-in camera (Scope Capture Digital Microscope, Guangdong, China) connected to an IBM compatible personal computer using a fixed magnification of 120x. The bitmap images were recorded with a resolution of $1280 \times 1024$ pixels per image. Digital microscope images were cropped to $350 \times 400$ pixels using a Microsoft office picture manager to specify/ standardize the area of roughness measurement.

The cropped images were analyzed using WS $\times$ M software (Version.5 develop 4.1, Nanotec, Electronica, SL). Within the WS $\times$ M software, all limits, sizes, frames and measured parameters are expressed in pixels. Therefore, system calibration was done to convert the pixels into absolute real world units. Calibration was accomplished by comparing an object of known size (a ruler in this study) with a scale generated by the software. Subsequently, a 3D image of the surface profile of 
the specimens was created. Three 3D images were collected for each specimen, both in the central area and in the sides at an area of $10 \mu \mathrm{m} \times 10 \mu \mathrm{m}$. WS $\times$ M software was used to calculate the average height in $\mu \mathrm{m}$ of every specimen. The average of the ten examined specimens was taken as the mean surface roughness of each group.

\section{Color stability (CS)}

Color stability testing was carried out according to the regulations of ISO 4049; $2009^{[51]}$. Specimens ( $n=11$ per group) were prepared, cured, polished and cleaned in the same way described for threebody wear and surface roughness testing. A specimen from each group was stored dry for 7 days at $37{ }^{\circ} \mathrm{C}$ in vacuum oven chambers (Vacuum drying chambers, Binder, Bohemia, North America) and served as the color reference. Other ten specimens were divided into two divisions $(n=5)$. In the first division, specimens were stored in distilled water for 7 days at $37^{\circ} \mathrm{C}$ to demonstrate which color changes arise as a result of water storage. While in the second division, each specimen was initially dried at $37{ }^{\circ} \mathrm{C}$ for $24 \mathrm{~h}$. Then, one half of each specimen was covered with tin foil and the whole specimen was stored in water at $37{ }^{\circ} \mathrm{C}$ in a xenon light box (SIEMENS Procmat Manual Dental Xenon Box, Germany). After $24 \mathrm{~h}$, the foil was removed and the specimen was dried for another 5 days at $37^{\circ} \mathrm{C}$.

The color of each specimen was measured using a portable reflective spectrophotometer (X-Rite, model RM200QC, Neu-Isenburg, Germany). A white background was chosen and the color evaluation was performed according to the CIE L*a*b* color space. The color changes $(\Delta \mathrm{E})$ of the specimens were evaluated using the following formula [52]:

$$
\Delta \mathbf{E}_{\text {CIELAB }}=\left(\Delta \mathrm{L}^{* 2}+\Delta \mathbf{a}^{* 2}+\Delta \mathbf{b}^{* 2}\right)^{1 / 2}
$$

Where: $L^{*}=$ lightness $(0-100), a^{*}=$ color change of the axis red/green and $b^{*}=$ color variation of the axis yellow/blue.
Thus, it was possible to compare the color change after immersion treatment by the $\Delta \mathrm{E}$ parameters of CIEL*a*b* system.

\section{Statistical analysis}

Statistical analysis was carried out using an IBM compatible personal computer with SPSS statistical package version 20 (SPSS Inc. Released 2011. Armnok, NY: IBM Corp.). One-way analysis of variance (ANOVA) and significance level of ( $p \leq$ 0.05 ) were applied for the statistical analysis of the data of the investigated properties. As there were significant differences between groups of cuspal flexure, microhardness, wear and surface roughness, Levene's test for homogeneity of variances was carried out for the data of each property ( $\mathrm{p} \leq$ $0.05)$ to choose the appropriate test for multiple comparisons. Equal variances were confirmed for data of microhardness $(\mathrm{p}=0.071)$, wear $(\mathrm{p}=$ $0.394)$ and surface roughness $(p=0.253)$; therefore the Tukey test was used to determine differences between groups of each property. As equal variances were not confirmed for data of cuspal flexure ( $\mathrm{p}=$ 0.002), Dunnett's T3 test was used to determine differences between groups.

\section{RESULTS}

\section{Characterization results:}

Scanning electron microscopy (SEM):

\section{Bis-GMA nanofibers:}

Figure 1 shows smooth surface of electrospun BisGMA nanofibers. The diameter of the synthesized nanofibers ranged between 70 and $100 \mathrm{~nm}$. Scanning was carried out at a magnification of $20000 \mathrm{x}$.

\section{UDMA nanofibers}

Similarly, at a magnification of 20000 x, Figure 2 shows SEM image of electrospun UDMA nanofibers. The nanofibers diameter ranged from 50 to100 $\mathrm{nm}$. 
Fourier transform infrared spectroscopy (FTIR):

\section{Bis-GMA nanofibers}

In Figure 3, formation of mixed organic nanofibers (Bis-GMA/TEGDMA/PEGDMA) is indicated by the IR spectra of the aromatic (BisGMA) and aliphatic (TEGDMA/ PEGDMA) compounds which displayed intense peaks at 3457 $\mathrm{cm}^{-1}$ due to O-H stretching, at 2965- $2873 \mathrm{~cm}^{-1}$ due to $\mathrm{C}-\mathrm{H}$ stretching of $\mathrm{CH}_{2}$, at 1608 and 830$810 \mathrm{~cm}^{-1}$ due to $\mathrm{C}=\mathrm{C}$ stretching, at $1509 \mathrm{~cm}^{-1}$ due to $\mathrm{C}-\mathrm{C}$ stretching, at $1245-1100 \mathrm{~cm}^{-1}$ due to $\mathrm{C}-\mathrm{O}-\mathrm{C}$ stretching and at $1450 \mathrm{~cm}^{-1}$ due to $\mathrm{C}=\mathrm{O}$ stretching. In addition, IR spectra displayed peaks at 1600-1625 $\mathrm{cm}^{-1}$ due to benzene ring stretching in Bis-GMA.

\section{UDMA nanofibers:}

In case of UDMA nanofibers, the FTIR, as shown in Figure 4, displays specific absorption bands attributed to urethane NH (3500-3320 $\left.\mathrm{cm}^{-1}\right)$, the vibrations of the $\mathrm{CH}_{2}$ groups $\left(2870-2950 \mathrm{~cm}^{-1}\right)$ and carbonyl unit (CO) from the urethane, as well as to the ester moieties $\left(1717 \mathrm{~cm}^{-1}\right)$. The absorption bands for carbon-carbon double bond on the methacrylate function can be detected at 1608 and $810 \mathrm{~cm}^{-1}$, the $\mathrm{C}-\mathrm{C}$ vibration at $1535-1509 \mathrm{~cm}^{-1}$, the C-O-C unit in the region $1100-1245 \mathrm{~cm}^{-1}$, at 1368 $\mathrm{cm}^{-1}$ due to $\mathrm{C}-\mathrm{H}$ stretching in $\mathrm{CH}_{3}$ and at $1450 \mathrm{~cm}^{-1}$ due to $\mathrm{C}=\mathrm{O}$ stretching.

\section{Results of investigated properties:}

Means and standard deviations of cuspal flexure (CF), Vickers microhardness (HV), three-body wear (W), surface roughness (SR) and color stability (CS) of all studied groups are listed in Table 2. Results of multiple comparison between groups (Tukey test and Dunnett's T3 test) are shown in Table 2 as well.

Statistical analysis revealed significant differences for cuspal flexure ( $p=0.001)$, microhardness $(p=0.001)$, wear $(p=0.001)$ and surface roughness $(\mathrm{p}=0.001)$ but not for color stability $(\mathrm{p}=0.068)$.

All reinforced groups (with both Bis-GMA and UDMA nanofibers) showed lower cuspal flexure (CF), lower microhardness (HV) and greater wear (W) than the control group. In both categories of

TABLE (2) Means and standard deviations (in parentheses) of cuspal flexure, Vickers microhardness, threebody wear, surface roughness and color stability. Each value represents the mean of ten specimens. Different superscript letters indicates statistically significant differences between groups of each column $(\mathrm{p} \leq 0.05)$.

\begin{tabular}{|c|c|c|c|c|c|c|}
\hline \multirow{2}{*}{$\begin{array}{c}\text { Type of } \\
\text { nanofibers }\end{array}$} & $\begin{array}{c}\text { Percentage of } \\
\text { nanofibers }(\%)\end{array}$ & $\begin{array}{c}\text { Cuspal Flexure } \\
(\%)\end{array}$ & $\begin{array}{c}\text { Vickers } \\
\text { Microhardness } \\
(\text { VHN })\end{array}$ & $\begin{array}{c}\text { Three-body Wear } \\
(\mu \mathrm{m})\end{array}$ & $\begin{array}{c}\text { Surface } \\
\text { Roughness }(\mu \mathrm{m})\end{array}$ & $\begin{array}{c}\text { Color Stability } \\
(\Delta \mathrm{E})\end{array}$ \\
\hline \multirow{2}{*}{ None } & $\begin{array}{c}\text { Control group } \\
(0 \%)\end{array}$ & $0.352(0.012)^{\mathrm{f}}$ & $75.7(4.029)^{\mathrm{a}}$ & $2.07(0.0161)^{\mathrm{d}}$ & $0.151(0.009)^{\mathrm{a}}$ & $0.516(0.084)^{\mathrm{a}}$ \\
\hline \multirow{3}{*}{$\begin{array}{c}\text { Bis-GMA } \\
\text { nanofibers }\end{array}$} & $7 \%$ & $0.284(0.030)^{\mathrm{a}}$ & $73.2(4.104)^{\mathrm{a}, \mathrm{c}}$ & $2.44(0.331)^{\mathrm{a}}$ & $0.150(0.004)^{\mathrm{a}, \mathrm{c}}$ & $0.482(0.062)^{\mathrm{a}}$ \\
\cline { 2 - 8 } & $11 \%$ & $0.265(0.017)^{\mathrm{a}, \mathrm{b}}$ & $72.2(5.245)^{\mathrm{a}, \mathrm{d}}$ & $3.16(0.211)^{\mathrm{b}}$ & $0.144(0.007)^{\mathrm{a}, \mathrm{d}}$ & $0.517(0.101)^{\mathrm{a}}$ \\
\hline \multirow{3}{*}{$\begin{array}{c}\text { UDMA } \\
\text { nanofibers }\end{array}$} & $11 \%$ & $0.223(0.022)^{\mathrm{c}}$ & $69.5(5.380)^{\mathrm{a}, \mathrm{f}}$ & $3.83(0.203)^{\mathrm{c}}$ & $0.139(0.007)^{\mathrm{c}, \mathrm{d}, \mathrm{f}}$ & $0.562(0.087)^{\mathrm{a}}$ \\
\cline { 2 - 8 } & $15 \%$ & $0.214(0.010)^{\mathrm{c}}$ & $68.1(8.233)^{\mathrm{b}, \mathrm{c}, \mathrm{d}, \mathrm{f}, \mathrm{f}, \mathrm{i}}$ & $3.94(0.281)^{\mathrm{c}}$ & $0.135(0.007)^{\mathrm{b}, \mathrm{d}, \mathrm{i}}$ & $0.536(0.117)^{\mathrm{a}}$ \\
\cline { 2 - 8 } & $20 \%$ & $0.202(0.009)^{\mathrm{c}}$ & $64.6(6.022)^{\mathrm{b}, \mathrm{d}, \mathrm{f}, \mathrm{i}}$ & $4.54(0.328)^{\mathrm{e}}$ & $0.124(0.009)^{\mathrm{b}}$ & $0.568(0.096)^{\mathrm{a}}$ \\
\hline
\end{tabular}


reinforced groups, within the range studied $(7-20$ wt \%), there was a systematic decrease in $\mathrm{CF}$ and $\mathrm{HV}$ and a systematic increase in $\mathrm{W}$ mean values with increasing the percent of nanofibers. For a given percent, groups reinforced with UDMA nanofibers exhibited slightly lower CF and HV and higher $\mathrm{W}$ results than those reinforced with BisGMA nanofibers.

All reinforced groups recorded lower surface roughness (SR) than the control group. UDMA nanofibers showed comparable values to that of BisGMA nanofibers at $7 \%$ and $11 \%$ percents but better

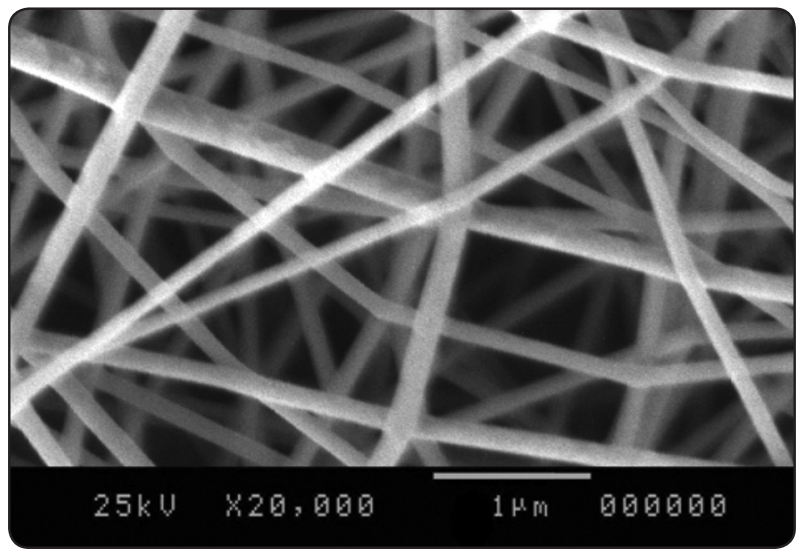

Fig. (1) Showing SEM image $(20,000 \mathrm{x})$ of electrospun BisGMA nanofibers (diameter: 70-100 nm).

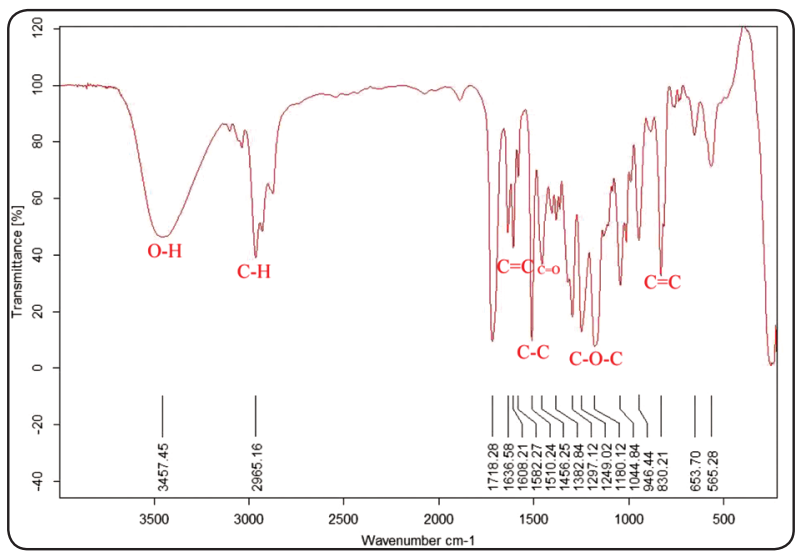

Fig. (3) Showing the FTIR spectra of electrospun BisGMA nanofibers made up of (Bis-GMA/TEGDMA/ PEGDMA). values at $15 \%$ and $20 \%$. Within the range studied (7 - $20 \mathrm{wt} \%)$, for both types, there was an inverse relationship between the increased SR and the percent of added nanofibers. Despite the absence of statistically significant differences between the investigated groups $(\mathrm{p}=0.068)$, with the exception of the group reinforced with 7\% Bis-GMA nanofibers, all groups recorded slightly higher color change $(\Delta \mathrm{E})$ than the control one. For both types of nanofibers, there was no clear correlation between the percent of reinforcing nanofibers and the value of $\Delta \mathrm{E}$ in all studied groups.

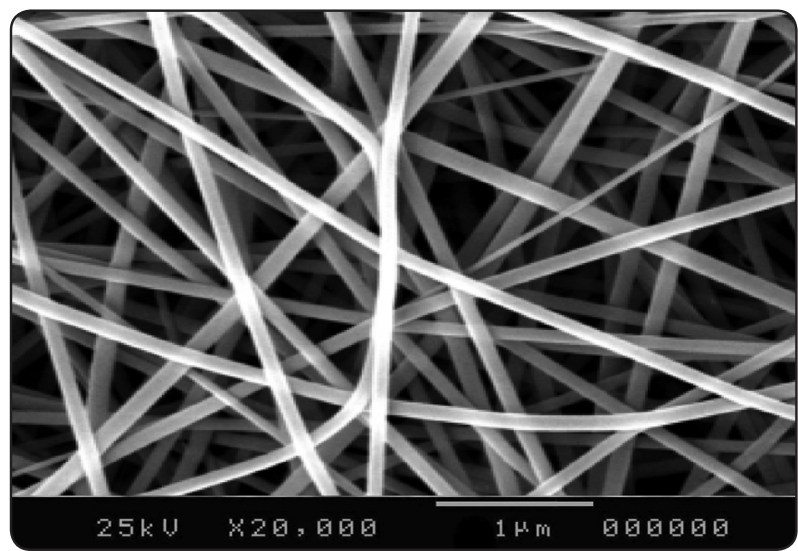

Fig. (2) Showing SEM image $(20,000$ x) of electrospun UDMA nanofibers (diameter: 50-100 nm).

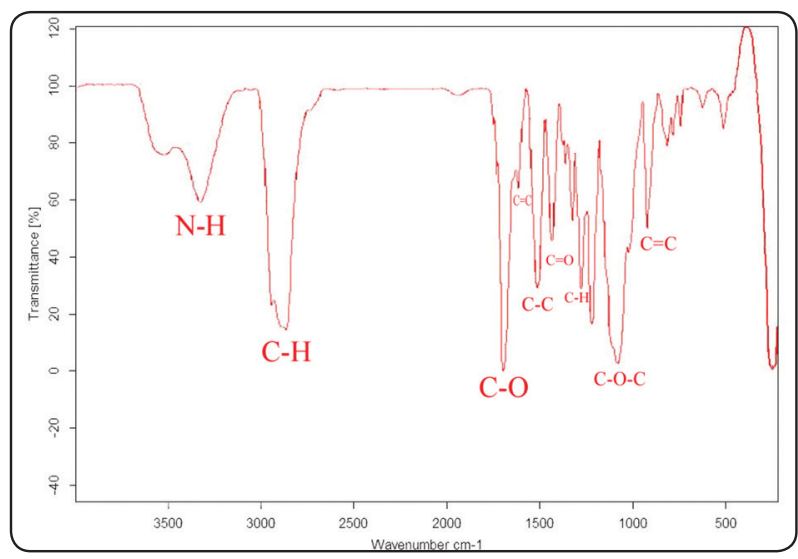

Fig. (4) Showing the FTIR spectra of electrospun UDMA nanofibers made up of (UDMA/ PEGDMA). 


\section{DISCUSSION}

Reinforcing resin-composite restorative materials with nanofibers synthesized from the same monomers used in the resin matrix of such materials is advantageous. Some of the advantages of using such nanofibers were explained in details in a previous study conducted by the authors ${ }^{[42]}$. The enhancing effect of reinforcing nanofibers has been reported to be primarily related to the length, form, orientation and quantity of fibers in the resin matrix. Similarly critical, is the impregnation of these fibers with and adhesion to the resin matrix ${ }^{[52]}$.

Though the positive impact of such nanofibers, either organic or inorganic, on resin-composite materials is more visible in certain properties such as flexural strength and fracture toughness, there are many other properties to be evaluated when applying such reinforcing nanofibers. Therefore, in the present study, we tried to find out what effect these nanofibers would have on cuspal flexure (CF), Vickers microhardness (HV), three-body wear (W), surface roughness (SR) and color stability (CS) of these restoratives. The rationale of choosing a ratio of 7-15 wt \% of Bis-GMA and 7-20 wt\% of UDMA nanofibers for reinforcing studied experimental resin-composites groups as well as why these nanofibers were roughly termed Bis-GMA and UDMA nanofibers though other components such as TEGDMA and PEGDMA were included in the initial mix used to produce these nanofibers was stated in the previous study ${ }^{[42]}$.

As the statistical analysis revealed significant differences between studied groups in case of CF, HV, W and SR but not in case of CS, the first null hypothesis was partly rejected. All reinforced groups (with both types of nanofibers) exhibited significantly lower CF than the control group. More incorporation of nanofibers (within the range studied, 7-20 wt\%) to the experimental resincomposite produced a systematic decrease in CF. As one of the means proposed to reduce polymerization shrinkage of resin-based restoratives, addition of a percent of pre-polymerized, even partially, resin (in the form of organic nanofibers) can result in decreasing the overall polymerization contraction of the mass. This is because incorporation of prepolymerized resin fillers decreases the volume fraction of the polymerizable resin and increases the filler volume fraction resulting in reduction of the polymerization shrinkage ${ }^{[53]}$. However, in our study, incorporation of a percent of pre-polymerized resin in the form of nanofibers was at the expense of the inorganic filler particles, which means that the resin fraction in all experimental groups was the same as the control group, in addition to the replaced resin part in the form of nanofibers. Despite this, the nanofibers-reinforced groups recorded lower $\mathrm{CF}$ than the control group. This may be attributed to the shape, length and orientation of the reinforcing nanofibers in the resin matrix. Theoretically, the reinforcing effect of fiber fillers is majorly based on stress transfer from the weaker polymer matrix to the fillers ${ }^{[54]}$. This can be effectively achieved when having fiber length equal to or greater than the critical fiber length $\left(\mathrm{L}_{\mathrm{C}}\right)$ that was reported to range between 0.5 and $1.6 \mathrm{~mm}$ in Bis-GMA based polymer matrix ${ }^{[55]}$. Once the reinforcing fibers attain a critical length, most of the resin-composite properties are improved with increasing the fibers aspect ratio (length to diameter). As the aspect ratio increases, strengths (flexural, tensile, fatigue), modulus and fracture toughness are remarkably increased. In addition, the overall polymerization shrinkage is reduced, particularly along the fiber axis with less shrinkage stress ${ }^{[56]}$.

At a given percent, groups reinforced with UDMA nanofibers recorded slightly lower CF than those reinforced with Bis-GMA nanofibers. This result could be explained based on the inherent properties, such as viscosity and degree of conversion, of the two main resin monomers forming the nanofibers (Bis-GMA and UDMA). As UDMA possesses far lower viscosity $(\eta=23 \mathrm{~Pa})$ compared to Bis-GMA $(\eta=1000-1200 \mathrm{~Pa}){ }^{[57]}$ even after being diluted, it shows more conversion upon exposure 
to the high voltage $(20 \mathrm{Kv})$ and heat accompanying the electrospinning process. Better conversion of the double bond to single bond in case of UDMA nanofibers compared to Bis-GMA nanofibers, before incorporation into the experimental resin-composite, minimized the contraction stress, and hence the net $\mathrm{CF}$, of the whole mass upon photopolymerization.

In agreement with our findings, Wang et al. ${ }^{[58]}$, though using inorganic nanofibers $\left(\mathrm{SiO}_{2}\right.$ nanofibers $)$ to reinforce a resin-composite based on Bis-GMA/ TEGDMA, reported an effective reduction in the polymerization shrinkage compared to the $\mathrm{SiO}_{2}$ nanoparticles as well as the $\mathrm{SiO}_{2}$ microparticles. In addition, Garoushi et al. ${ }^{[52]}$ reported an improvement in the polymerization shrinkage stress upon using E-glass short fiber fillers for reinforcement of an experimental resin-composite.

Unfavorably, results of $\mathrm{HV}$ and $\mathrm{W}$ of the reinforced groups were poorer than that of the control group. $\mathrm{VH}$ of reinforced groups was significantly lower but $\mathrm{W}$ was significantly greater than that recorded for the control group. HV of reinforced groups decreased systematically with more addition of nanofibers (of both types) with slightly better results for Bis-GMA reinforced groups, at a given percent. For W, there was a direct relationship between increase of $\mathrm{W}$ values of reinforced groups and percent of added nanofibers of both types. Generally speaking, besides creep deformation, wear is a major factor in the observed loss of height and other changes that take place in the occlusal surface of resin-composite restorations ${ }^{[59]}$. Intra-orally, three-body wear occurs as a result of presence of food stuff between occlusal surfaces as well as toothpastes during tooth brushing. In resincomposite restorations, it involves loss of resin matrix between relatively harder inorganic filler particles and subsequent dislodgement of projecting filler particles. Filler loading, size and distribution were reported to be very critical in protecting resin matrix and enhancing the wear resistance of a resincomposite material ${ }^{[60]}$. In a given period, the amount of material removed during in vivo performance was reported to be dependent on several factors such as the type of material, type of restoration, manipulative procedures and patient's eating habits [61].

When the percent is equal, groups reinforced with UDMA nanofibers exhibited slightly greater $\mathrm{W}$ than those reinforced with Bis-GMA nanofibers. The relatively better results ( $\mathrm{HV}$ and $\mathrm{W}$ ) of BisGMA nanofibers compared to the UDMA nanofibers could be explained on the basis that Bis-GMA monomer possesses more enhanced mechanical properties than UDMA ${ }^{[62,63]}$. Lower HV and poorer W resistance of the reinforced groups compared to the control one could be attributed to the nature and characteristics of the reinforcing nanofibers. Because of the organic nature of Bis-GMA and UDMA nanofibers, they possess far lower surface mechanical properties than conventional inorganic (particulate/fiber) fillers. Opposite to our findings, when $\mathrm{SiO}_{2}$ (inorganic) nanofibers were used in experimental resin-composite, they showed an enhanced wear resistance. Authors ${ }^{[58]}$ attributed this improvement to the ability of incorporated inorganic nanofibers to cover other micro/nano-sized particulate fillers preventing their dislodgement during wearing cycles. In our study, however, as the reinforcing nanofibers are organic in nature, they worn away more easily compared to the inorganic filler particles in the control group.

Surface roughness (SR) and wear (W) of a restorative material are intimately related to one another. In addition to plaque accumulation, gingival irritation, staining and poor aesthetics, a restoration with rough surface shows a greater rate of material loss because of abrasive wear [64]. Interestingly, our study showed that the SR of the reinforced groups was significantly lower than that of the control group. Such a positive result could be reasoned on the basis that the polymeric reinforcing nanofibers are strongly cross-linked with the resin matrix forming a homogenous matrix-filler complex. Such a complex, when subjected to wearing cycles, both the resin matrix and reinforcing fibers exhibited 
equal and simultaneous loss of material maintaining the smoothness of its surface. More incorporation of nanofibers of both types was accompanied by lower SR with slight superiority to groups reinforced with UDMA nanofibers over those reinforced with Bis-GMA nanofibers, when the ratio is equal. Lower consistency and simpler structure of UDMA monomer compared to Bis-GMA monomer may explain this difference.

On the contrary to the previous four investigated properties, there were no significant differences between color stability (CS) of studied groups. For groups reinforced with UDMA nanofibers, there was no consistent trend of the results where $11 \mathrm{wt} \%$ recorded greater color change $(\Delta \mathrm{E})$ than that recorded by higher or lower percents. Mixing of added nanofibers with the resin matrix and specimen finishing and polishing may have a role in this result. In case of Bis-GMA nanofibers, however, there was a slightly greater $\Delta \mathrm{E}$ with increasing the percent of reinforcing fibers. $\mathrm{CS} / \Delta \mathrm{E}$ of a resin-based material was said to be influenced by intrinsic and extrinsic factors. Intrinsic factors are related to the material itself such as the monomer type and its hydrophobicity, polymerization type, photoinitiator and filler type. In a completely polymerized material, intrinsic factors have been reported to play a minor role in the material's color change. Extrinsic discoloration caused by foods and beverages through absorption and adsorption represents the main factor influencing CS of a resincomposite ${ }^{[65,66]}$. Overall, interestingly, $\Delta \mathrm{E}$ recorded in our study for all groups was far lower than the clinically acceptable value $(\leq 3.3)^{[67,68]}$.

Because of having a central repeating ethoxy group that shows high affinity to water molecule through hydrogen bonding to oxygen, TEGDMA is known with its hydrophilic properties ${ }^{[69]}$. Addition of a percent of TEGDMA to Bis-GMA, as a diluent, causes the resin-composite based on Bis-GMA to exhibit higher hydrophilicity and increased water sorption than that based on UDMA monomer [70]. Resin-composites with high water sorption and hydrophilicity were said to be more susceptible to discoloration as colorants are likely absorbed with water into the resin matrix ${ }^{[71]}$. In the current study, absence of a big difference of $\Delta \mathrm{E}$ between Bis-GMA and UDMA nanofibers-reinforced groups may be attributed to the smaller percents added (7$20 \mathrm{wt} \%$ ) compared to the bulk of the resin matrix or the pre-polymerized nature and nanoscale of the added nanofibers that rendered water sorption by the material very minimal.

As our findings revealed statistical differences between the effect of Bis-GMA nanofibers and that of UDMA nanofibers on the investigated properties, therefore, the second null hypothesis was rejected. For CF and SR, UDMA nanofibers recorded relatively superior results than those showed by Bis-GMA nanofibers. This could be attributed to the simpler structure, better conversion and smaller diameter (50-100 nm) of UDMA nanofibers compared to Bis-GMA nanofibers (70-100 nm). In case of $\mathrm{HV}$ and $\mathrm{W}$, the reverse took place where groups reinforced with Bis-GMA showed relatively greater $\mathrm{HV}$ and better $\mathrm{W}$ resistance than those reinforced with UDMA nanofibers. This was attributed to the enhanced mechanical properties of Bis-GMA compared to UDMA. Both types of nanofibers, however, exhibited comparable $\Delta \mathrm{E}$ of studied resin-composite groups.

Addition of polymeric nanofibers (Bis-GMA and UDMA) significantly improved the CF and SR of the studied experimental resin-composite compared to the control group. While keeping the CS relatively unaffected, $\mathrm{HV}$ and $\mathrm{W}$ were detrimentally influenced, compared to the control group. A resincomposite material with such properties may be useful as a restorative material provided that being capped with a superficial layer of another resincomposite with more enhanced hardness and better wear resistance. This strongly supports the concept of "a bilayered composite restoration", where a resincomposite reinforced with such nanofibers could be used as a base layer and covered with a top layer of a resin-composite with more enhanced surface 
properties $^{[72]}$. Such a combination of the two layers in a bilayered resin-composite structure produces a biomimetic restoration system resembling that of dentin-enamel complex ${ }^{[73]}$.

\section{CONCLUSIONS}

- Addition of Bis-GMA and UDMA nanofibers, though maintaining the CS unchanged, significantly improved CF and SR of studied experimental resin-composite.

- Because of the negative impact of these nanofibers on $\mathrm{HV}$ and $\mathrm{W}$, such a reinforced resincomposite should be covered with a superficial layer of another material with more enhanced surface properties.

- Within the range of added nanofibers (7-20 wt $\%$ ), Bis-GMA nanofibers were superior to UDMA nanofibers in $\mathrm{HV}$ and $\mathrm{W}$ while the reverse took place in case of CF and SR. Both nanofibers recorded, however, comparable CS results.

\section{REFERENCES}

1. Peutzfeldt A. Resin composites in dentistry: the monomer systems. Eur J Oral Sci 1997; 105:97-116.

2. Asmussen E, Peutzfeldt A. Influence of UEDMA BisGMA and TEGDMA on selected mechanical properties of experimental resin composites. Dent Mater 1998; 14:51-56.

3. Sideridou I, Tserki V, Papanastasiou G. Effect of chemical structure on degree of conversion in light-cured dimethacrylate-based dental resins. Biomat 2002; 23:1819-1829.

4. Lovell LG, Stansbury JW, Syrpes DC, Bowman CN. Effects of composition and reactivity on reaction kinetics of dimethacrylate/dimethacrylate copolymerizations. Macromol 1999; 32:3913-3921.

5. Khatri CA, Stansbury JW, Schultheisz CR, Antonucci JM. Synthesis, characterization and evaluation of urethane derivatives of Bis-GMA. Dent Mater, 2003; 19:584-588.

6. Braem M, Finger W, Van Doren VE, Lambrechts P, Vanherle G. Mechanical Properties and Filler Fraction of Dental Composites. Dent Mater, 1989; 5:346-348

7. Munksgaard EC, Hansen EK, Kato H. Wall-to-Wall Polymerization Contraction of Composite Resins Versus Filler Content. Scand J of Dent Res, 1987; 95:526-531.
8. Arikawa H, Kanie T, Fujii K, Takahashi H, Ban S. Effect of Filler Properties in Composite Resins on Light Transmittance Characteristics and Color. Dent Mater J, 2007; 26:38-44

9. Goldberg AJ, Burstone CJ. The Use of Continuous Fiber Reinforcement in Dentistry. Dent Mater, 1992; 8:197-202.

10. Garoushi S, Vallittu PK, Lassila LV. Short Glass Fiber Reinforced Restorative Composite Resin with Semi-Inter Penetrating Polymer Network Matrix. Dent Mater, 2007; 23:1356-1362.

11. Freilich MA, Karmaker AC, Burstone CJ, Goldberg AJ. Development and Clinical Applications of a Light-Polymerized Fiber-Reinforced Composite. J Prosthet Dent, 1998; 80:311-318.

12. Garoushi S, Säilynoja E, Vallittu PK, Lassila L. Physical properties and depth of cure of a new short fiber reinforced composite. Dent Mater 2013;29:835-841.

13. Fráter M, Forster A, Keresztúri M, Braunitzer G, Nagy K.In vitro fracture resistance of molar teeth restored with ashort fibre-reinforced composite material. J Dent 2014;42:1143-1150.

14. Vallittu PK. High-aspect ratio fillers: Fiber-reinforced composites and their anisotropic properties. Dent Mater 2015: 31: 1-7.

15. Venugopal J, Ramakrishna S: Applications of polymer nanofibers in biomedicine and biotechnology, Appl Bioch and Biotech 2005; 125: 147-157.

16. Cipitria A, Skelton A, Dargaville T, Dalton P and Hutmacher D. Design, fabrication and characterization of PCL electrospun scaffolds - a review. J Mater Chem 2011; 21: 9419-9453.

17. Leung V, Ko F. Biomedical applications of nanofibers. Polym Adv Technol 2011; 22:350-365.

18. Ko F, Gogotsi Y, Ali A, Naguib N, Ye H, Yang G, et al. Electrospinning of continuous carbon nanotube-filled nanofiber yarns. Adv Mater 2003; 15:1161-1165.

19. Braga RR, Ballester RY, Ferracane JL. Factors involved in the development of polymerization shrinkage stress in resin-composites: a systematic review, Dent Mater, 2005; 21:962-970.

20. Braga RR, Ferracane JL, Condon JR. Polymerization contraction stress in dual-cure cements and its effect on interfacial integrity of bonded inlays. J Dent 2002; 30:333-340.

21. Kleverlaan CJ, Feilzer AJ. Polymerization shrinkage and contraction stress of dental resin composites. Dent Mater. 2005; 21:1150-1157. 
22. Giachetti L, Russo DS, Bambi C, Grandini R. A Review of Polymerization Shrinkage Stress: Current Techniques for Posterior Direct Resin Restorations. J Contemp Dent Pract, 2006; 7:079-088.

23. Labella R, Lambrechts P, Van Meerbeek B, Vanherle G. Polymerization Shrinkage and Elasticity of Flowable Composites and Filled Adhesives. Dent Mater, 1999; 15:128-137.

24. Ferracane JL. Correlation between hardness and degree of conversion during the setting reaction of unfilled dental restorative resins. Dent Mater 1985; 1:11-14.

25. Leprince JG, Palin WM, Vanacker J, Sabbagh J, Devaux J, Leloup G. Physico-mechanical characteristics of commercially available bulk-fill composites. J Dent 2014; 42:9931000 .

26. Aguiar FH, Braceiro AT, Ambrosano G, Lovadino JR. Hardness and diametral tensile strength of a hybrid composite resin polymerized with different modes and immersed in ethanol or distilled water media. Dent Mater 2005; 21:10981103 .

27. Wassell RW, McCabe JF, Walls AWG. Subsurface Deformation Associated with Hardness Measurements of Composites. Dent Mater, 1992; 8:218-223.

28. El-Safty S, Akhtar R, Silikas N, Watts DC. Nanomechanical properties of dental resin-composites. Dent Mater, 2012; 28: 1292-1300.

29. Yesil ZD, Alapati S, Johnston W, Seghi RR. Evaluation of the wear resistance of new nanocomposite resin restorative materials. J Prosthet Dent 2008; 99:435-443.

30. Palaniappan S, Bharadwaj D, Mattar DL, Peumans M, van Meerbeek B, Lambrechts P. Three-year randomized clinical trial to evaluate the clinical performance and wear of a nanocomposite versus a hybrid composite. Dent Mater 2009; 25:1302-1314.

31. Condon JR, Ferracane JL. Factors effecting dental composite wear in vitro. J Biomed Mater Res (Applied Biomaterials) $1997 ; 38: 303-313$.

32. Hu X, Shortall AC, Marquis PM. Wear of three dental composites under different testing conditions. J Oral Rehab 2002;29:756-764.

33. Hu X, Marquis PM, Shortall AC. Influence of filler loading on the two-body wear of a dental composite. J Oral Rehab 2003;30:729-737.
34. Koottathape N, Takahashi H, Iwasaki N, Kanehira M, Finger WJ. Two- and three-body wear of composite resins. Dent Mater 2012; 28:1261-1270.

35. Morgan M. Finishing and polishing of direct posterior resin restorations. Pract Proced Aesthet Dent 2004; 16:211-277.

36. Yap AU, Lye KW, Sau CW. Surface characteristics of toothcolored restoratives polished utilizing different polishing systems. Oper Dent 1997; 22:260-265.

37. Quirynen M, Bollen CM, Papaioannou W, Van Eldere J, van Steenberghe D. The influence of titanium abutment surface roughness on plaque accumulation and gingivitis: short term observations. Inter J Oral Maxillofac Impl 1996; 11:169-178.

38. Bollenl CML, Lambrechts P, Quirynen M. Comparison of surface roughness of oral hard materials to the threshold surface roughness for bacterial plaque retention: a review of the literature. Dent Mater 1997; 13:258-269.

39. Arocha MA, Mayoral JR, Lefever D, Mercade M, Basilio J, Roig M. Color stability of siloranes versus methacrylatebased composites after immersion in staining solutions. Clin Oral Investig 2013;17:1481-1487.

40. Dietschi D, Campanile G, Holz J, Meyer JM. Comparison ofthe color stability of ten new-generation composites: an in vitro study. Dent Mater 1994; 6:353-362.

41. Ertas E, Guler AU, Yucel AC, Koprulu H, Guler E. Color stability of resin composites after immersion in differente drinks. Dent Mater J 2006; 25:371-376.

42. El-Safty SM, Kenawy ER. Synthesis of Bis-GMA and UDMA Nanofibers for Reinforcing Experimental resincomposites: Influence on Degree of Conversion, Depth of Cure, Flexural Strength, Flexural Modulus and Fracture Toughness. Egypt Dent J, 2019; 65:863-880.

43. Do T, Church B, Veríssimo C, Hackmyer SP, Tantbirojn D, Simon JF et al. Cuspal flexure, depth-of-cure, and bond integrity of bulk-fill composites. Pediat Dent. 2014; 36: 468473.

44. Moorthy A, Hogg CH, Dowling AH, Grufferty BF, Benetti AR, Fleming GJ. Cuspal deflection and microleakage in premolar teeth restored with bulk-fill flowable resin-based composite base materials. J Dent. 2012; 40(6): 500-505.

45. ASTM E-384. "Standard test method for microhardness of materials". American Society for Testing and Materials ASTM, Annual Book of Standards, v. 3; 1999. 
46. ISO/TS 14569-2 standard (Dental Materials - Guidance on testing of wear - Part 2: Wear by two - and/or three-body contact, 2001.

47. De Gee AJ, Pallav P, Davidson CL. Effect of abrasion medium on wear of stress-bearing composites and amalgam in vitro. J Dent Res, 1986; 65:654-658.

48. Condon JR, Ferracane JL. Evaluation of composite wear with a new multi-mode oral wear simulator. Dent Mater, 1996; 12:218-226.

49. Bates JF, Stafford GD, Harrison A (1975). Masticatory function - a review of the literature. 1 . The form of the masticatory cycle. J Oral Rehabil, 1975; 2:281-301.

50. International Organization for Standardization. Geometrical Product Specification (GPS) - Surface Texture: Area - Part 2: Terms, Definitions, Surface Texture Parameters and Techniques. 2012; ISO 25178:2012 (2).

51. International Organization for Standardization. Dentistrypolymer-based filling, restorative and luting materials 2009; ISO 4049:2009 (1).

52. Garoushi S, Vallittu PK, Watts DC, Lassila LVJ. Polymerization shrinkage of experimental short glass fiber-reinforced composite with semi-inter penetrating polymer network matrix. Dent Mater 2008; 24:211-215.

53. Al Sunbul H, Silikas N, Watts DC. Polymerization shrinkage kinetics and shrinkage-stress in dental resin-composites. Dent Mater 2016; 32:998-1006.

54. Vallittu PK, Lassila VP, Lappalainen R. Transverse strength and fatigue of denture acrylic-glass fiber composite. Dent Mater 1994; 10:116-121.

55. Cheng TH, Jones FR, Wang D. Effect of fiber conditioning on the interfacial shear strength of glass-fiber composite. Compos Sci Technol 1993; 48:89-96.

56. Petersen RC. Discontinuous fiber-reinforced composites above critical length. J Dent Res 2005; 84:365-370.

57. Barszczewska-Rybarek IM. Structure-property relationships in dimethacrylate networks based on Bis-GMA, UDMA and TEGDMA. Dent Mater 2009; 25:1082-1089.

58. Wang X, Cai Q, Zhang X, Wei Y, Xu M, Yang X, Ma Q, Cheng Y, Deng X. Improved performance of Bis-GMA/ TEGDMA dental composites by net-like structures formed from $\mathrm{SiO} 2$ nanofiber fillers. Mater Sci and Eng C, 2016; 59:464-470.

59. Oden A, Ruyter IE, Oysaed H. Creep and Recovery of Composites for Use in Posterior Teeth During Static and Dynamic Compression. Dent Mater, 1988; 4:147-150.
60. Bayne S, Thompson J, Swift J, Stamatiades P, Wilkerson M. A characterisation of first-generation flowable composites. J Am Dent Assoc, 1998; 129:567-577.

61. Sulong, MZ, Aziz, RA. Wear of materials used in dentistry: a review of the literature. J Prosthet Dent, 1990; 63:342-349.

62. Asmussen E. Restorative resins; hardness and strength vs. quantity of remaining double bonds. Scand J Dent Res 1982; 90: 484-489.

63. Ferracane JL, Greener EH. The effect of resin formulation on the degree of conversion and mechanical properties of dental restorative resins. J Biomed Mater Res, 1986; 20: 121-131.

64. Jefferies, SR. Abrasive finishing and polishing in restorative dentistry: a state-of-the-art review. Dent Clin North Am, 2007; 51:379-397.

65. Bagheri R, Burrow MF, Tyas M. Influence of food-simulating solutions and surface finish on susceptibility to staining of aesthetic restorative materials. J Dent 2005; 33:389-398.

66. Shintani H, Satou N, Yukihiro A, Satou J, Yamane I, Kouzai $\mathrm{T}$, et al. Water sorption, solubility and staining properties of microfilled resins polished by various methods. Dent Mater J 1985; 4:54-62.

67. Ren YF, Feng L, Serban D, Malmstrom HS. Effects of common beverage colorants on color stability of dental composite resins: the utility of a thermo-cycling stain challenge model in vitro. J Dent 2012; 40 Suppl 1:e48-56.

68. Ruyter IE, Nilner K, Moller B. Color stability of dental composite resin materials for crown and bridge veneers. Dent Mater 1987; 3:246-51.

69. Arima T, Hamada T, McCabe JF. The effects of cross-linking agents on some properties of HEMA-based resins. J Dent Res 1995; 74:1597-1601.

70. Kalachandra S, Turner DT. Water sorption of polymethacrylate networks: bis-GMA/TEGDM copolymers. Journal of Biomedical Materials Research 1987; 21:329-338.

71. Fontes ST, Fernandez MR, de Moura CM, Meireles SS. Color stability of a nanofill composite: effect of different immersion media. J Appl Oral Sci 2009; 17:388-391.

72. Vallittu PK. An overview of development and status of fiber-reinforced composites as dental and medical biomaterials. Acta Biomat Odonto Scand, 2018; 4:44-55.

73. Frese C, Decker C, Rebholz J, et al. Original and repair bond strength of fiber-reinforced compsites in vitro. Dent Mater. 2014; 30:456-462. 